\title{
TOPOLOGICAL SYMMETRY GROUPS OF GRAPHS IN 3-MANIFOLDS
}

\author{
ERICA FLAPAN AND HARRY TAMVAKIS \\ (Communicated by Daniel Ruberman)
}

\begin{abstract}
We prove that for every closed, connected, orientable, irreducible 3-manifold there exists an alternating group $A_{n}$ which is not the topological symmetry group of any graph embedded in the manifold. We also show that for every finite group $G$ there is an embedding $\Gamma$ of some graph in a hyperbolic rational homology 3 -sphere such that the topological symmetry group of $\Gamma$ is isomorphic to $G$.
\end{abstract}

\section{INTRODUCTION}

Characterizing the symmetries of a molecule is an important step in predicting its behavior. Chemists often model a molecule as a graph in $\mathbb{R}^{3}$. They define the point group of a molecule as the group of isometries of $\mathbb{R}^{3}$ which take the molecular graph to itself. This is a useful way of representing the symmetries of a rigid molecule. However, molecules which are flexible or partially flexible may have symmetries which are not induced by isometries of $\mathbb{R}^{3}$. Jon Simon 20] introduced the concept of the topological symmetry group in order to study the symmetries of such nonrigid molecules. This group has not only been used to study the symmetries of such molecules but also the non-rigid symmetries of any graph embedded in $S^{3}$. In this paper we extend this study to graphs embedded in other 3-manifolds.

In 1938, Frucht [6] showed that every finite group is the automorphism group of some connected graph. By contrast, in [5] we proved that the only finite simple groups which can occur as the topological symmetry group of a graph embedded in $S^{3}$ are cyclic groups and the alternating group $A_{5}$. Thus, we can say that automorphism groups of connected graphs are universal for finite groups, while topological symmetry groups of graphs embedded in $S^{3}$ are not. We now prove that topological symmetry groups of graphs embedded in any given closed, connected, orientable, irreducible 3 -manifold are not universal for finite groups.

In particular, let $M$ be a 3-manifold, let $\gamma$ be an abstract graph, and let $\Gamma$ be an embedding of $\gamma$ in $M$. Note that by a graph we shall mean a finite, connected graph with at most one edge between any pair of vertices and two distinct vertices for every edge. The topological symmetry group $\operatorname{TSG}(\Gamma, M)$ is defined to be the subgroup of the automorphism group $\operatorname{Aut}(\gamma)$ consisting of those automorphisms of $\gamma$ which are induced by a homeomorphism of the pair $(M, \Gamma)$. Allowing only

Received by the editors June 2, 2011 and, in revised form, August 3, 2011.

2010 Mathematics Subject Classification. Primary 57M15, 57M60; Secondary 05C10, 05C25.

The first author was supported in part by NSF Grant DMS-0905087.

The second author was supported in part by NSF Grant DMS-0901341. 
orientation-preserving homeomorphisms defines the orientation-preserving topological symmetry group $\operatorname{TSG}_{+}(\Gamma, M)$. We prove the following result.

Theorem 1.1. For every closed, connected, orientable, irreducible 3-manifold $M$, there exists an alternating group $A_{n}$ which is not isomorphic to $\operatorname{TSG}(\Gamma, M)$ for any graph $\Gamma$ embedded in $M$.

On the other hand, it follows from Frucht's theorem [6] that every finite group $G$ can occur as the topological symmetry group of a graph embedded in some closed 3 -manifold (which depends on $G$ ). We prove the following stronger result.

Theorem 1.2. For every finite group $G$, there is an embedding $\Gamma$ of a graph in a hyperbolic rational homology 3 -sphere $M$ such that $\operatorname{TSG}(\Gamma, M) \cong G$.

A graph is said to be 3 -connected if at least 3 vertices must be removed together with the edges they contain in order to disconnect the graph or reduce it to a single vertex. In [5], we showed that for every 3 -connected graph $\Gamma$ embedded in $S^{3}$, there is a subgroup of $\operatorname{Diff}_{+}\left(S^{3}\right)$ isomorphic to $\operatorname{TSG}_{+}\left(\Gamma, S^{3}\right)$. We now prove this is not the case for all 3-manifolds.

Theorem 1.3. For every closed, orientable, irreducible, 3-manifold $M$ that is not Seifert fibered, there is an embedding of a 3-connected graph $\Gamma$ in $M$ such that $\mathrm{TSG}_{+}(\Gamma, M)$ is not isomorphic to any subgroup of $\operatorname{Diff}_{+}(M)$.

\section{Proof of Theorem 1.1}

We begin by introducing some notation. Let $\Gamma$ be an embedding of a graph in a 3-manifold $M$. Let $V$ denote the set of embedded vertices of $\Gamma$, and let $E$ denote the set of embedded edges of $\Gamma$. We construct a neighborhood $N(\Gamma)$ as the union of two sets, $N(V)$ and $N(E)$, which have disjoint interiors. In particular, for each vertex $v \in V$, let $N(v)$ denote a ball around $v$ whose intersection with $\Gamma$ is a star around $v$, and let $N(V)$ denote the union of all of these balls. For each embedded edge $e \in E$, let $N(e)$ denote a solid cylinder $D^{2} \times I$ whose core is $e-N(V)$, such that $N(e) \cap \Gamma \subseteq e$, and $N(e)$ meets $N(V)$ in a pair of disks. Let $N(E)$ denote the union of all the solid cylinders $N(e)$. Let $N(\Gamma)=N(V) \cup N(E)$. We shall use $\partial^{\prime} N(e)$ to denote the annulus $\partial N(\Gamma) \cap N(e)$ in order to distinguish it from the sphere $\partial N(e)$.

By the standard smoothing results in dimension 3 (proved in [14]), if a particular automorphism of an embedded graph $\Gamma$ can be induced by an orientation-preserving homeomorphism of $(M, \Gamma)$, then the same automorphism can be induced by an orientation-preserving homeomorphism of $(M, \Gamma)$ which is a diffeomorphism except possibly on the set of vertices of $\Gamma$. Thus we shall abuse notation and call such a homeomorphism a diffeomorphism of $(M, \Gamma)$.

Lemma 2.1. Let $\Gamma$ be a graph embedded in a ball $B \subseteq M$, where $M$ is an orientable, irreducible 3-manifold, and suppose that $\mathrm{TSG}_{+}(\Gamma, M)$ is a non-abelian simple group. Then $\mathrm{TSG}_{+}(\Gamma, M) \cong A_{5}$.

Proof. If $M=S^{3}$ the result follows from [5]. Thus we assume $M \neq S^{3}$. We obtain an embedding of $\Gamma$ in $S^{3}$ by gluing a ball to the outside of $B$. Since $\Gamma$ is connected, $S^{3}-\Gamma$ is irreducible. Thus $M-\Gamma$ is the connected sum of the irreducible manifolds $M$ and $S^{3}-\Gamma$. Hence the splitting sphere $\partial B$ is unique up to isotopy in $M-\Gamma$. Thus 
$\mathrm{TSG}_{+}(\Gamma, M)$ is induced by a group $G$ of orientation-preserving diffeomorphisms of $(M, \Gamma)$ that takes $B$ to itself.

Since the restriction of $G$ to $B$ extends radially to $S^{3}$ taking $\Gamma$ to itself, it follows that $\operatorname{TSG}_{+}(\Gamma, M) \leq \operatorname{TSG}_{+}\left(\Gamma, S^{3}\right)$. We show containment in the other direction as follows. Let $p$ be a point of $S^{3}$ which is disjoint from $\Gamma$. Let $g$ be an orientation-preserving diffeomorphism of the pair $\left(S^{3}, \Gamma\right)$. We can compose $g$ with an isotopy to obtain an orientation-preserving diffeomorphism $h$ of the pair $\left(S^{3}, \Gamma\right)$ which pointwise fixes a neighborhood of $p$ disjoint from $\Gamma$ and induces the same automorphism of $\Gamma$ as $g$. It follows that $\operatorname{TSG}_{+}\left(\Gamma, S^{3}\right)$ can be induced by a group $G^{\prime}$ of orientation-preserving diffeomorphisms of $\left(S^{3}, \Gamma\right)$ which take the ball $B$ to itself fixing its boundary pointwise. We may therefore restrict the elements of $G^{\prime}$ to $B$ and then extend them to $M$ by the identity. It follows that $\operatorname{TSG}_{+}(\Gamma, M)=$ $\mathrm{TSG}_{+}\left(\Gamma, S^{3}\right)$, and hence by [5] we have $\operatorname{TSG}_{+}(\Gamma, M) \cong A_{5}$.

Lemma 2.2. Let $\Gamma$ be a graph embedded in a closed, orientable, irreducible 3manifold $M$ such that $\mathrm{TSG}_{+}(\Gamma, M)$ is a non-abelian simple group. Then there is a graph $\Lambda$ embedded in $M$ with $\mathrm{TSG}_{+}(\Lambda, M) \cong \mathrm{TSG}_{+}(\Gamma, M)$ such that $\partial N(\Lambda)-$ $\partial^{\prime} N(E)$ is incompressible in $\mathrm{Cl}(M-N(\Lambda))$.

Proof. The proof of this lemma is similar to the proof of a stronger result [5, Prop. 2] for $M=S^{3}$. We will therefore give only the main ideas of the argument, with many of the details omitted.

We say that $\Gamma$ has a separating ball $B$ if $B$ meets $\Gamma$ in a single vertex $v$ with valence at least 3 and $\operatorname{Int}(B)$ and $M-B$ each have non-empty intersection with $\Gamma$. In this case, we say that $B \cap \Gamma$ is a branch of $\Gamma$ at $v$.

Suppose that $\partial N(\Gamma)-\partial^{\prime} N(E)$ is compressible in $\mathrm{Cl}(M-N(\Gamma))$. Then there is a sphere in $M$ meeting $\Gamma$ in a single point such that each complementary component of the sphere intersects $\Gamma$ non-trivially. Since $M$ is irreducible, one of these components is a ball. Also, $\Gamma$ cannot be homeomorphic to an arc because $\operatorname{TSG}_{+}(\Gamma, M)$ is nonabelian. Thus $\Gamma$ has a separating ball at some vertex $v$.

First suppose, for the sake of contradiction, that $\operatorname{TSG}_{+}(\Gamma, M)$ fixes $v$ but does not setwise fix some branch $\Gamma_{1}$ at $v$. Let $\left\{\Gamma_{1}, \ldots, \Gamma_{n}\right\}$ denote the orbit of $\Gamma_{1}$ under $\mathrm{TSG}_{+}(\Gamma, M)$. Now the action of $\mathrm{TSG}_{+}(\Gamma, M)$ on $\left\{\Gamma_{1}, \ldots, \Gamma_{n}\right\}$ defines a non-trivial monomorphism $\Phi: \operatorname{TSG}_{+}(\Gamma, M) \rightarrow S_{n}$. To see that $\Phi$ is onto, let $(i j)$ be a transposition in $S_{n}$. Since $\Gamma_{i}$ and $\Gamma_{j}$ are in the orbit of $\Gamma_{1}$ under $\operatorname{TSG}_{+}(\Gamma, M)$, we can choose a pair of separating balls $B_{i}$ and $B_{j}$ which are disjoint except at $v$ such that there is an orientation-preserving diffeomorphism $g$ of $(M, \Gamma)$ with $g\left(\left(B_{i}, \Gamma_{i}\right)\right)=\left(B_{j}, \Gamma_{j}\right)$. We can then choose a separating ball $E$ containing $B_{i} \cup B_{j}$ such that $\partial E \cap\left(B_{i} \cup B_{j}\right)=\{v\}$. Now define an orientation-preserving diffeomorphism $h:\left(E, \Gamma_{i} \cup \Gamma_{j}\right) \rightarrow E$ such that $h\left|B_{i}=g\right| B_{i}, h\left|B_{j}=g^{-1}\right| B_{j}$, and $h \mid \partial E$ is the identity. Finally, extend $h$ to $M-E$ by the identity. Thus we have $h:(M, \Gamma) \rightarrow(M, \Gamma)$ and $h$ induces $(i j)$ on $\Gamma$, and therefore $\Phi$ is onto. Since $\mathrm{TSG}_{+}(\Gamma, M)$ is simple and non-abelian, this is impossible.

Let $m$ denote an integer which is larger than the number of vertices in $\Gamma$. We will show that there is another graph $\Gamma^{\prime}$ in $M$ such that $\operatorname{TSG}_{+}\left(\Gamma^{\prime}, M\right) \cong \operatorname{TSG}_{+}(\Gamma, M)$ and $\Gamma^{\prime}$ has fewer (possibly 0 ) branches at $v$. By repeating this argument as necessary, one eventually obtains the embedded graph $\Lambda$. By the above paragraph, we only need to consider the following two cases. 
Case 1. $\mathrm{TSG}_{+}(\Gamma, M)$ fixes $v$ and setwise fixes every branch of $\Gamma$ at $v$.

Since $\operatorname{TSG}_{+}(\Gamma, M)$ is non-trivial, there is some branch $\Gamma_{1}$ at $v$ on which $\mathrm{TSG}_{+}(\Gamma, M)$ acts non-trivially. Let $e_{1}$ denote an edge in $\Gamma_{1}$ containing $v$, and let $\left\{e_{1}, \ldots, e_{r}\right\}$ be the orbit of $e_{1}$ under $\operatorname{TSG}_{+}(\Gamma, M)$. We define $\Gamma^{\prime}$ as $\Gamma_{1}$ together with $m$ vertices of valence 2 added to the interior of each $e_{i}$. We can define a nontrivial monomorphism $\psi: \mathrm{TSG}_{+}(\Gamma, M) \rightarrow \mathrm{TSG}_{+}\left(\Gamma^{\prime}, M\right)$ since each automorphism of $\Gamma$ induces an automorphism of $\Gamma^{\prime}$. Because of the $m$ vertices on each $e_{i}$, every automorphism of $\Gamma^{\prime}$ fixes $v$. It is not hard to check that $\psi$ is onto, and $\Gamma^{\prime}$ has fewer branches at $v$ than $\Gamma$.

Case 2. $v$ is not fixed by $\operatorname{TSG}_{+}(\Gamma, M)$.

Let $\left\{\Gamma_{1}, \ldots, \Gamma_{n}\right\}$ denote the orbit of $\Gamma_{1}$ under $\operatorname{TSG}_{+}(\Gamma, M)$. Let $e_{1}$ denote an edge of the graph $\operatorname{cl}\left(\Gamma-\left(\Gamma_{1} \cup \cdots \cup \Gamma_{n}\right)\right)$ that contains $v$, and let $\left\{e_{1}, \ldots, e_{r}\right\}$ and $\left\{v_{1}, \ldots, v_{q}\right\}$ denote the orbits of $e_{1}$ and $v$ respectively under $\operatorname{TSG}_{+}(\Gamma, M)$. Now define $\Gamma^{\prime}$ as $\operatorname{cl}\left(\Gamma-\left(\Gamma_{1} \cup \cdots \cup \Gamma_{n}\right)\right)$ together with $m$ vertices of valence 2 added to the interior of each $e_{i}$. We can define a non-trivial monomorphism $\psi: \mathrm{TSG}_{+}(\Gamma, M) \rightarrow$ $\mathrm{TSG}_{+}\left(\Gamma^{\prime}, M\right)$ since each automorphism of $\Gamma$ induces an automorphism of $\Gamma^{\prime}$. Since $\mathrm{TSG}_{+}\left(\Gamma^{\prime}, M\right)$ cannot be cyclic, $\Gamma^{\prime}$ cannot be a simple closed curve. Thus every automorphism of $\Gamma^{\prime}$ leaves $\left\{v_{1}, \ldots, v_{q}\right\}$ setwise invariant. Now it is not hard to check that $\psi$ is onto and $\Gamma^{\prime}$ has fewer branches at $v$ than $\Gamma$.

In addition to Lemmas 2.1 and 2.2 our proof of the next result will employ Jaco-Shalen [8] and Johannson's [9] theory of characteristic splittings along tori and annuli. For a survey of the definitions and statements, see [1].

Proposition 2.3. Let $M$ be a closed, connected, orientable, irreducible 3-manifold. Then there exists an integer $d$ depending only on $M$ such that for any graph $\Gamma$ embedded in $M$, if $\operatorname{TSG}_{+}(\Gamma, M)$ is a non-abelian simple group, then the order of $\mathrm{TSG}_{+}(\Gamma, M)$ is at most $d$.

Proof. We shall assume that $\Gamma$ is not contained in a ball in $M$, since otherwise the result follows from Lemma 2.1. Since the proof is lengthy we divide it into six steps.

Step 1 . We determine the number $d$.

Apply the Characteristic Submanifold Theorem [8, 9] to $M$ to obtain a minimal collection $\Theta$ of incompressible tori in $M$ such that the closure of each component of $M-\Theta$ is either atoroidal or Seifert fibered, and $\Theta$ is unique up to isotopy. If $\Theta$ is non-empty, let $C_{1}, \ldots, C_{m}$ denote the closures of the components of $M-\Theta$; otherwise let $m=1$ and $C_{1}=M$. Now we consider two cases.

Case 1. Either $\Theta$ is non-empty or $\Theta$ is empty and $M$ is Seifert fibered.

Consider a component $C_{i}$ which is Seifert fibered (possibly $C_{i}=M$ ). We see as follows that there is an upper bound $n_{i}$ on the order of all finite simple non-abelian groups which can act faithfully on the base space of the fibration of $C_{i}$. If the base space is a sphere or projective plane (possibly with holes), then $n_{i}=60$ is an upper bound since $A_{5}$ is the only finite simple non-abelian group which can act faithfully on a sphere or projective plane. If the base space has negative Euler characteristic $\chi$, then $n_{i}=84|\chi|$ is an upper bound by a classical result of Hurwitz [7]. Since no finite simple non-abelian groups can act faithfully on a torus or Klein bottle (possibly with holes), if the base space is one of these surfaces we let $n_{i}=1$. 
Now consider a component $C_{i}$ which is not Seifert fibered. By the hypotheses of this case $C_{i} \neq M$. It follows that $C_{i}$ is atoroidal and has non-empty incompressible boundary. Hence, by Thurston's Hyperbolization Theorem [21], $C_{i}$ admits a complete, hyperbolic structure with totally geodesic boundary. Now by Mostow's Rigidity Theorem [18, there is an integer $n_{i}$ such that no finite group of diffeomorphisms of $C_{i}$ has order greater than $n_{i}$.

After having chosen an $n_{i}$ associated with each component $C_{i}$, we let $d=$ $\operatorname{Max}\left\{n_{1}, \ldots, n_{m}, m !, 60\right\}$.

Case 2. $\Theta$ is empty and $M$ is not Seifert fibered.

By the Geometrization Theorem [15, 16, 17, $M$ has a geometric structure. Also since $M$ is irreducible and not Seifert fibered, $M$ does not admit a circle action. Furthermore, by the Elliptization Theorem [16], a 3-manifold with finite fundamental group is elliptic and hence Seifert fibered. Thus $M$ has infinite $\pi_{1}$, no circle action, and is irreducible. Hence by Kojima [11] there is a bound $q$ on the order of finite groups of diffeomorphisms of $M$. In this case, we let $d=\operatorname{Max}\{q, 60\}$.

Now let $\Gamma$ be a graph embedded in $M$ such that $\operatorname{TSG}_{+}(\Gamma, M)$ is a simple non-abelian group. By Lemma 2.2. without loss of generality, we can assume that $\partial N(\Gamma)-\partial^{\prime} N(E)$ is incompressible in $\mathrm{Cl}(M-N(\Gamma))$. We will prove that order $\left(\mathrm{TSG}_{+}(\Gamma, M)\right) \leq d$.

Step 2. We choose $W \subseteq \mathrm{Cl}(M-N(\Gamma))$ and a group $G$ of diffeomorphisms of $(M, \Gamma)$ leaving $W$ setwise invariant.

Since $\Gamma$ is connected and not contained in a ball, and $M$ is irreducible, the manifold $\mathrm{Cl}(M-N(\Gamma))$ is irreducible. Thus we can apply the Characteristic Submanifold Theorem [8, 9] to the manifold $\mathrm{Cl}(M-N(\Gamma))$ to get a minimal family of characteristic tori. When we split $\mathrm{Cl}(M-N(\Gamma))$ along these tori, $\partial N(\Gamma)$ is contained entirely in one component, the closure of which we denote by $X$. Since $\partial N(\Gamma)-\partial^{\prime} N(E)$ is incompressible in $\mathrm{Cl}(M-N(\Gamma)), \Gamma$ has no valence one vertices. Thus since $\mathrm{TSG}_{+}(\Gamma, M)$ is not cyclic, it follows that $\Gamma$ contains at least two simple closed curves. Therefore the genus of $\partial N(\Gamma)$ is at least two, and hence $X$ cannot be Seifert fibered. It follows that $X$ is atoroidal, and since $M$ is irreducible, $X$ is also irreducible.

Let $P$ denote the set of annuli in $\partial^{\prime} N(E)$ together with the torus boundary components of $X$. Since $\partial N(\Gamma)-\partial^{\prime} N(E)$ is incompressible in $\mathrm{Cl}(M-N(\Gamma)), \partial X-P$ is incompressible in $X$. Thus we can now apply the Characteristic Submanifold Theorem for Pared Manifolds [8, 9] to the pared manifold $(X, P)$. Since $X$ is atoroidal, this gives us a minimal family $\Omega$ of incompressible annuli in $X$ with boundaries in $\partial X-P$ such that if $W$ is the closure of any component of $X-\Omega$, then the pared manifold $(W, W \cap(P \cup \Omega))$ is either simple, Seifert fibered, or $I$ fibered, and the set $\Omega$ is unique up to isotopy.

Let $G$ denote the collection of orientation-preserving diffeomorphisms of $(M, \Gamma)$ which leave $X, \Omega, N(V)$, and $N(E)$ setwise invariant. It follows from the uniqueness up to isotopy of each of these sets that every automorphism in $\operatorname{TSG}_{+}(\Gamma, M)$ is induced by some element of $G$. Suppose that two elements of $G$ induce the same automorphism on $\Gamma$. Then they induce the same permutation on the set of components of $\partial N(V)$. Since $\Gamma$ has at most one edge between two vertices and every edge has two distinct vertices, they also induce the same permutation on the set of 
components of $\partial^{\prime} N(E)$. It now follows that they induce the same permutation on the set of annuli in $\Omega$ as well as on the components of $X-\Omega$.

We construct a graph $\lambda$ associated with $(X, \Omega)$ by representing the closure of each component of $X-\Omega$ by a vertex and defining an edge between a pair of vertices if the components they represent are adjacent. Observe that each annulus in $\Omega$ can be capped off by a pair of disks in $N(V)$ to obtain a sphere in $M$. Since $M$ is irreducible, any sphere in $M$ separates. It follows that all of the annuli in $\Omega$ separate $X$. Thus $\lambda$ is a tree. Now $G$ induces a group of automorphisms on $\lambda$ which either fixes a vertex or fixes an edge setwise. Suppose that no vertex of $\lambda$ is fixed by $G$. Then some edge of $\lambda$ is inverted by an element of $G$. Hence there is an annulus $A \in \Omega$ which is setwise invariant under $G$ and some element of $G$ which interchanges the components adjacent to $A$. Thus we can define a non-trivial homomorphism $\varphi: \operatorname{TSG}_{+}(\Gamma, M) \rightarrow \mathbb{Z}_{2}$, where $\varphi(a)=1$ if and only if $a$ is induced by an element of $G$ which interchanges the components adjacent to $A$. However this is impossible since $\operatorname{TSG}_{+}(\Gamma, M)$ is simple and non-abelian. Hence there must be a vertex of $\lambda$ which is fixed by $G$.

If the action of $G$ on $\lambda$ is non-trivial, then we choose a particular fixed vertex $w$ of $\lambda$ which is adjacent to a vertex which is not fixed by $G$. In this case, let $W$ be the closure of the component of $X-\Omega$ represented by $w$. Then $W$ is setwise fixed by $G$ and some annuli in $W \cap \Omega$ are not setwise fixed by $G$. Observe that all of the components of $W \cap \Omega$ are contained in a single component of $\partial W$. If this component of $\partial W$ were a torus, then there would be a non-trivial homomorphism from $\mathrm{TSG}_{+}(\Gamma, M)$ to $\mathbb{Z}_{r}$, where $r$ is the number of annuli in $W \cap \Omega$. Since $\operatorname{TSG}_{+}(\Gamma, M)$ is a finite simple non-abelian group, this component of $\partial W$ cannot be a torus.

If the action of $G$ on $\lambda$ is trivial, we choose $W$ to be the closure of some component of $X-\Omega$ such that some components of $\partial N(V) \cap W$ are permuted by $G$. We know there is such a component of $X-\Omega$ since $\operatorname{TSG}_{+}(\Gamma, M)$ is non-trivial and $\partial N(\Gamma)$ is contained in $X$. Observe that since the annuli of $\Omega$ separate $X$, and $\partial N(\Gamma)$ is connected, all of the components of $W \cap \partial N(V)$ are contained in a single component of $\partial W$. As above, if the component of $\partial W$ containing $W \cap \partial N(V)$ were a torus, then there would be a non-trivial homomorphism from $\operatorname{TSG}_{+}(\Gamma, M)$ to a finite cyclic group. Thus again, this component $\partial W$ is not a torus.

Since not all of the components of $\partial W$ are tori, $W$ cannot be Seifert fibered. Thus the pared manifold $(W, W \cap(P \cup \Omega))$ is either $I$-fibered or simple.

Step 3. We prove that the pared manifold $(W, W \cap(P \cup \Omega))$ is simple.

Suppose, for the sake of contradiction, that $(W, W \cap(P \cup \Omega))$ is $I$-fibered. Then there is an $I$-bundle map of $W$ over a surface such that $W \cap(P \cup \Omega)$ is the preimage of the boundary of the surface. It follows that the corresponding $\partial I$-bundle is $\partial N(V) \cap$ $W$ and has either one or two components. If $\partial N(V) \cap W$ has two components which are interchanged by some element of $G$, then there would be a non-trivial homomorphism from $\operatorname{TSG}_{+}(\Gamma, M)$ to $\mathbb{Z}_{2}$. Thus we can assume that each component of $\partial N(V) \cap W$ is setwise invariant under $G$. It now follows from our definition of $W$ that some annulus $F_{1}$ in $\Omega \cap W$ is not setwise invariant under $G$.

Let $\left\{F_{1}, \ldots, F_{r}\right\}$ denote the orbit of $F_{1}$ under $G$. Since $r>1$, the boundary components of $F_{1}$ do not co-bound an annulus in $\partial N(V)-W$. Thus we can cap off $F_{1}$ in $\partial N(V)-W$ to obtain a sphere. Let $E_{1}$ denote the closure of the component of the complement of this sphere in $M$ which is disjoint from $W$. The orbit of $E_{1}$ under $G$ is a pairwise disjoint collection $E_{1}, \ldots, E_{r}$ such that each $F_{i} \subseteq \partial E_{i}$. Suppose 
that $E_{1}$ is not a ball. Since $M$ is irreducible, $\mathrm{Cl}\left(M-E_{1}\right)$ is a ball containing $E_{2}$. But since $E_{2} \cong E_{1}$ is not a ball, this is impossible. Thus each $E_{i}$ must be a ball.

Now the action of $G$ on the orbit $\left\{F_{1}, \ldots, F_{r}\right\}$ defines a non-trivial monomorphism $\Phi: \mathrm{TSG}_{+}(\Gamma, M) \rightarrow S_{r}$. Furthermore, since $\mathrm{TSG}_{+}(\Gamma, M)$ is non-abelian, $r>2$. Hence the base surface of the $I$-bundle has at least three boundary components. We see as follows that $\Phi$ is onto. Let $(i j)$ be a transposition in $S_{r}$. Then there is a $g \in G$ such that $g\left(F_{i}\right)=F_{j}$. We saw above that each component of $\partial N(V) \cap W$ is setwise invariant under $G$. Hence a boundary component of $F_{i}$ and its image under the element $g$ are in the same component of $\partial N(V) \cap W$, and they project to distinct boundary components of the base surface of the $I$-bundle. Let $N$ denote a regular neighborhood in the base surface of these two boundary components together with an arc between them. Then $N$ is a disk with two holes. Now since $M$ is orientable, the preimage of $N$ in the $I$-bundle is a product $N \times I$. We add the balls $E_{i}$ and $E_{j}$ to $N \times I$ along the annuli $F_{i}$ and $F_{j}$ respectively to obtain a solid cylinder $C \times I$.

We will define a homeomorphism $h:(M, \Gamma) \rightarrow(M, \Gamma)$ as follows. Let $h\left|E_{i}=g\right| E_{i}$ and $h\left|E_{j}=g^{-1}\right| E_{j}$. Then extend $h$ within $N \times I$ so that $h$ restricted to the cylinder $\partial C \times I$ is the identity and $h$ leaves each of the disks $C \times\{0\}$ and $C \times\{1\}$ setwise invariant. Next we cap off the solid cylinder $C \times I$ in $N(V)$ to obtain a ball or pinched ball $B$ whose boundary intersects $\Gamma$ in either one or two vertices. Then extend $h$ within $B$ in such a way that $h \mid \partial B$ is the identity and $h$ leaves $\Gamma \cap B$ setwise invariant. Finally, we extend $h$ to $M-B$ by the identity. Now by our construction, $h:(M, \Gamma) \rightarrow(M, \Gamma)$ is an orientation-preserving homeomorphism such that $\Phi(h)=$ $(i j)$. It follows that $\Phi$ is onto. However, this is impossible since $\operatorname{TSG}_{+}(\Gamma, M)$ is a simple non-abelian group. Thus the pared manifold $(W, W \cap(P \cup \Omega))$ cannot be I-fibered, and hence must be simple.

Step 4 . We define a group of isometries $K$ of $W$ and prove $K \cong \operatorname{TSG}_{+}(\Gamma, M)$.

Since $W$ is simple, it follows from Thurston's Hyperbolization Theorem for Pared Manifolds [21] applied to $(W, W \cap(P \cup \Omega))$ that $W-(W \cap(P \cup \Omega))$ admits a finite volume complete hyperbolic metric with totally geodesic boundary. Let $D$ denote the double of $W-(W \cap(P \cup \Omega))$ along its boundary. Then $D$ is a finite volume hyperbolic manifold, and every element of $\operatorname{TSG}_{+}(\Gamma, M)$ is induced by an element of $G$ whose restriction to $W$ can be doubled to obtain a diffeomorphism of $D$. Now by Mostow's Rigidity Theorem [18, each such diffeomorphism of $D$ is homotopic to an orientation-preserving finite order isometry that restricts to an isometry of $W-(W \cap(P \cup \Omega))$. Furthermore, the set of all such isometries generates a finite group $K$ of isometries of $W-(W \cap(P \cup \Omega))$. By removing horocyclic neighborhoods of the cusps of $W-(W \cap(P \cup \Omega))$, we obtain a copy of the pair $(W, W \cap(P \cup \Omega))$ which is contained in $W-(W \cap(P \cup \Omega))$ and is setwise invariant under the isometry group $K$. We shall abuse notation and consider $K$ to be a finite group of isometries of $(W, W \cap(P \cup \Omega))$. Also $K$ induces a finite group of isometries of the tori and annuli in $W \cap(P \cup \Omega)$ with respect to a flat metric. In particular, $\partial N(V) \cap W$, $\partial^{\prime} N(E) \cap W$, and $\Omega \cap W$ are each setwise invariant under $K$. Finally, it follows from Waldhausen's Isotopy Theorem [23] that each element of $K$ is isotopic to an element of $G$ restricted to $W$ by an isotopy leaving $W \cap(P \cup \Omega)$ setwise invariant.

We show as follows that $\operatorname{TSG}_{+}(\Gamma, M) \cong K$. Let $a \in \operatorname{TSG}_{+}(\Gamma, M)$ be induced by the elements $g_{1}, g_{2} \in G$. Then, as we observed in Step 2, $g_{1}$ and $g_{2}$ induce the same 
permutation of the components of $\partial N(V) \cap W, \partial^{\prime} N(E) \cap W$, and $\Omega \cap W$. Now $g_{1} \mid W$ and $g_{2} \mid W$ are isotopic to some $f_{1}, f_{2} \in K$ by isotopies leaving $W \cap(P \cup \Omega)$ setwise invariant. Thus $f_{1}$ and $f_{2}$ also induce the same permutation of the components of $\partial N(V) \cap W, \partial^{\prime} N(E) \cap W$, and $\Omega \cap W$. Recall that the component of $\partial W$ which meets $N(V)$ is not a torus. Thus there is some component $J$ of $\partial N(V) \cap W$ which has $r \geq 3$ boundary components $\alpha_{1}, \ldots, \alpha_{r}$. Now $f_{1}(J)=f_{2}(J)$ and $f_{1}\left(\alpha_{i}\right)=f_{2}\left(\alpha_{i}\right)$ for each $i=1, \ldots, r$. Hence $f_{1}^{-1} \circ f_{2}$ restricts to a finite order diffeomorphism of $J$ which setwise fixes each component of $\partial J$. Since $J$ is a sphere with at least three holes, this implies that $f_{1}^{-1} \circ f_{2} \mid J$ is the identity. Finally, since $f_{1}$ and $f_{2}$ are isometries of $W$ which are identical on the surface $J \subseteq \partial W$, it follows that $f_{1}=f_{2}$. Thus the automorphism $a \in \mathrm{TSG}_{+}(\Gamma, M)$ determines a unique element of $K$, and hence there is a well-defined homomorphism $\Phi: \operatorname{TSG}_{+}(\Gamma, M) \rightarrow K$. Since every element of $K$ came from such an element of $\operatorname{TSG}_{+}(\Gamma, M), \Phi$ is onto. Now since $\mathrm{TSG}_{+}(\Gamma, M)$ is simple, it follows that $\mathrm{TSG}_{+}(\Gamma, M) \cong K$.

Step 5 . We extend $K$ to a set $W_{2}$ whose boundaries are spheres that do not bound balls in $M-W_{2}$ and tori that are not compressible in $M-W_{2}$.

Every annulus in $W \cap(P \cup \Omega)$ separates $X$ into two components. It follows that for any $\partial N(v)$ which meets $W$, each component of $\partial N(v)-W$ is either a disk or an annulus. Let $V_{1}$ denote the set of vertices of $\Gamma$ such that each component of $N\left(V_{1}\right)$ meets $W$ and let $E_{1}$ denote the set of edges such that each component of $N\left(E_{1}\right)$ meets $W$. We extend $K$ to $\partial N\left(V_{1}\right)-W$ as follows. Extend each element of $K$ radially within the disk components of $\partial N\left(V_{1}\right)-W$. Next consider an annulus component $A$ of $\partial N\left(V_{1}\right)-W$. The boundaries of $A$ must also be the boundary components of an annulus $A^{\prime}$ in $W \cap(P \cup \Omega)$. Since $K$ restricts to a finite group of isometries of $A^{\prime}$, we can extend $K$ to a finite group of isometries of $A$. In this way we have extended $K$ so that it is defined on each sphere in $\partial N\left(V_{1}\right)$. Now we extend $K$ radially within each of the balls in $N\left(V_{1}\right)$ and in $N\left(E_{1}\right)$. Thus we have extended $K$ to a finite group $K_{1} \cong K$ acting faithfully on $W_{1}=W \cup N\left(V_{1}\right) \cup N\left(E_{1}\right)$.

The boundary of $W_{1}$ consists of spheres and tori made up of the union of annuli in $\Omega \cap W$ with disks and annuli in $\partial N(V)-W$, together with the tori components of $\partial X \cap W$. Let $\left\{T_{1}, \ldots, T_{q}\right\}$ denote the tori components of $\partial W_{1}$ which are compressible in $M-W_{1}$. Since the set $\left\{T_{1}, \ldots, T_{q}\right\}$ is setwise invariant under $G$, the set $\left\{T_{1}, \ldots, T_{q}\right\}$ must be setwise invariant under $K_{1}$ as well. Now we can choose a set of pairwise disjoint compressing disks $\left\{D_{1}, \ldots, D_{r}\right\}$ for $\left\{T_{1}, \ldots, T_{q}\right\}$ whose boundaries are setwise invariant under $K_{1}$. Note that depending on the action of $K$ on each $T_{i}$, we may have $r>q$. We add a product neighborhood of each $D_{i}$ to $W_{1}$ to obtain a manifold whose boundary contains more spheres than $\partial W_{1}$ but contains no tori which are compressible in $M-W_{1}$. We extend $K_{1}$ to these product neighborhoods by defining it radially within each parallel disk. Furthermore, for any boundary component of the union of $W_{1}$ together with these product neighborhoods which bounds a ball in $M-W_{1}$, we add that ball and extend $K_{1}$ radially within the ball. Thus we have extended $K_{1}$ to a finite group $K_{2} \cong K_{1}$ acting faithfully on the manifold $W_{2}$ consisting of $W_{1}$ together with these product neighborhoods and balls. Observe that all of the components of $\partial W_{2}$ are either spheres which do not bound a ball in $M-W_{2}$ or tori which are not compressible in $M-W_{2}$.

Step 6 . We prove order $\left(\operatorname{TSG}_{+}(\Gamma, M)\right)=\operatorname{order}(K) \leq d$ by considering 3 cases.

Case 1. Some component of $\partial W_{2}$ is a sphere $S$. 
Since $M$ is irreducible and $S$ does not bound a ball in $M-W_{2}, S$ must bound a ball $B$ containing $W_{2}$. Now any other sphere in $\partial W_{2}$ separates $M$ such that the component of the complement which is disjoint from $W_{2}$ is contained in $B$. Since $B$ is a ball, this component is also a ball. As this is contrary to our definition of $W_{2}$, all of the components of $\partial W_{2}$ other than $S$ must be tori. By gluing another ball to $B$ we obtain $S^{3}$ such that each of the tori in $\partial W_{2}$ bounds a (possibly trivial) knot complement in $S^{3}$ that is disjoint from $W_{2}$. We extend $K_{2}$ radially within the complementary ball. Then we replace each knot complement by a solid torus in such a way that we obtain a homology sphere and we can extend $K_{2}$ radially within the solid tori. In this way we get an isomorphic finite simple non-abelian group $K_{3}$ of orientation-preserving diffeomorphisms of a homology sphere. Now it follows from Zimmerman [24] that $K_{3} \cong A_{5}$. Thus $\operatorname{order}(K)=\operatorname{order}\left(K_{3}\right)=60 \leq d$.

Case 2. $\partial W_{2}$ has torus components but no spherical components.

Recall that every component of $\partial W_{2}$ is incompressible in $M-W_{2}$. Suppose that $T$ is a torus component of $\partial W_{2}$ which is compressible in $W_{2}$. Let $N$ be a product neighborhood of a compressing disk in $W_{2}$. By cutting $T$ along $\partial N \cap T$ and capping off with the two disks in $\partial N-T$, we obtain a sphere $S$. Since $T$ is incompressible in $M-W_{2}$, the component of $M-S$ which is disjoint from $W_{2}-N$ is not a ball. Thus the component of $M-S$ containing $W_{2}-N$ must be a ball. Hence the union of this ball and the neighborhood $N$ is a solid torus $V$ such that $\partial V=T$ and $W_{2} \subseteq V \subseteq M$.

Let $T_{1}, \ldots, T_{r}$ denote the boundary components of $W_{2}$, and suppose that every $T_{i}$ is compressible in $W_{2}$. Then, by the argument above, each $T_{i}$ bounds a solid torus $V_{i}$ such that $W_{2} \subseteq V_{i} \subseteq M$. Now $G$ induces an orientation-preserving finite action on the solid tori $V_{1}, \ldots, V_{r}$ taking meridians to meridians, up to isotopy. Since $G$ induces a finite action on the set of tori $\left\{T_{1}, \ldots, T_{r}\right\}$ on the level of homology, this means there is also a set of longitudes $\left\{\ell_{1}, \ldots, \ell_{r}\right\}$ which are setwise invariant under $G$ up to isotopy. Hence the action that $K_{2}$ induces on the set of tori $\left\{T_{1}, \ldots, T_{r}\right\}$ leaves the set of longitudes $\left\{\ell_{1}, \ldots, \ell_{r}\right\}$ setwise invariant up to isotopy.

We obtain a homology sphere $W_{3}=W_{2} \cup U_{1} \cup \cdots \cup U_{r}$ by gluing a solid torus $U_{i}$ along each boundary component $T_{i}$ of $W_{2}$ so that a meridian $\mu_{i}$ of $U_{i}$ is glued to the longitude $\ell_{i}$. Thus $K_{2}$ leaves the set of meridians $\left\{\mu_{1}, \ldots, \mu_{r}\right\}$ setwise invariant up to isotopy. Now since the action of $K_{2}$ on the set of tori $\left\{\partial U_{1}, \ldots, \partial U_{r}\right\}$ is finite, for some $q \geq r$, we can find a set of pairwise disjoint meridians $\left\{m_{1}, \ldots, m_{q}\right\}$ for the solid tori $\left\{U_{1}, \ldots, U_{r}\right\}$ which is setwise invariant under $K_{2}$. Now extend $K_{2}$ radially from the set of meridians $\left\{m_{1}, \ldots, m_{q}\right\}$ to a set of pairwise disjoint meridional disks for the solid tori $U_{1}, \ldots, U_{r}$. These meridional disks cut the solid tori $U_{1}, \ldots, U_{r}$ into a set of solid cylinders, and hence we can also extend $K_{2}$ radially within this set of solid cylinders. In this way we obtain a finite group $K_{3} \cong K_{2}$ acting faithfully on the homology sphere $W_{3}$. Thus it again follows from Zimmerman [24] that $K_{3} \cong A_{5}$, and hence $\operatorname{order}(K)=\operatorname{order}\left(K_{3}\right)=60 \leq d$.

Therefore, we can assume that some component of $\partial W_{2}$ is an incompressible torus in $M$. If a torus component of $\partial W$ is setwise fixed by $K_{2}$, then $K_{2}$ restricts to a faithful action of the torus, which is impossible since $K_{2}$ is a finite simple non-abelian group. Thus some incompressible boundary component of $\partial W_{2}$ has non-trivial orbit $\left\{T_{1}, \ldots, T_{q}\right\}$ under $K_{2}$. Now either each $T_{i}$ is isotopic to a torus in the characteristic family $\Theta$ or each $T_{i}$ is vertical in a closed up Seifert fibered component of $M-\Theta$. Suppose that each $T_{i}$ is isotopic to a torus in $\Theta$. Then 
without loss of generality we can assume that each $T_{i}$ is in $\Theta$. It follows that there is a non-trivial monomorphism from $K_{2}$ to $S_{m}$ (where $m$ is the number of tori in $\Theta)$. Hence $\operatorname{order}(K)=\operatorname{order}\left(K_{2}\right) \leq m ! \leq d$.

Thus we can assume that each $T_{i}$ is a vertical torus in a closed up Seifert fibered component of $M-\Theta$ and $T_{i}$ is not isotopic to a torus in $\Theta$. Let $C_{1}, \ldots, C_{r}$ denote all of the closed up Seifert fibered components of $M-\Theta$. The action of $K_{2}$ on the set $\left\{T_{1}, \ldots, T_{q}\right\}$ induces an action on the set $\left\{C_{1} \cap W_{2}, \ldots, C_{r} \cap W_{2}\right\}$, which in turn defines a homomorphism from $K_{2}$ to $S_{r}$. Since $K_{2}$ is simple, either this homomorphism is trivial or $\operatorname{order}(K)=\operatorname{order}\left(K_{2}\right) \leq r ! \leq m ! \leq d$.

Therefore, we can assume that the homomorphism is trivial, and hence the orbit $\left\{T_{1}, \ldots, T_{q}\right\}$ is contained in a single Seifert fibered component $C$. Now there is a non-trivial monomorphism from $K_{2}$ to $S_{q}$. Since $K_{2}$ is simple and non-abelian, $q \geq 5$. In particular, the Seifert fibered space $C \cap W_{2}$ is a Haken manifold with more that two boundary components. Now it follows from Waldhausen 22 that the fibration on $C \cap W_{2}$ is unique up to isotopy. Hence by Meeks and Scott [13, $C \cap W_{2}$ has a $K_{2}$-invariant fibration. Thus $K_{2}$ induces an action on the base surface of the fibration of $C \cap W_{2}$. Let $F$ be the base surface for the Seifert fibration of $C$, and let $F^{\prime}$ be the base surface for the Seifert fibration of $C \cap W_{2}$. Since the vertical tori $T_{i}$ are incompressible in $C$, none of the boundary components of $F^{\prime}$ bounds a disk in $F$. Furthermore, since there are at least five such tori, $\chi(F) \leq \chi\left(F^{\prime}\right)<0$. Note that since the action of $K_{2}$ on the set $\left\{T_{1}, \ldots, T_{q}\right\}$ is non-trivial, $K_{2}$ cannot take each fiber of $C \cap W_{2}$ to itself. Thus $K_{2}$ induces an isomorphic action on $F^{\prime}$. Now using Hurwitz [7, we have order $(K)=\operatorname{order}\left(K_{2}\right) \leq 84\left|\chi\left(F^{\prime}\right)\right| \leq 84|\chi(F)| \leq d$.

Case $3 . \partial W_{2}$ is empty.

In this case, $W_{2}$ is the 3 -manifold $M$. Suppose that the set of characteristic tori $\Theta$ is non-empty. Since $\Theta$ is unique up to isotopy, we can find an isotopic set of tori $\Theta^{\prime}$ which are setwise invariant under $K_{2}$. Thus there is a homomorphism from $K_{2}$ to $S_{m}$ (recall that $m$ is the number of tori in $\Theta$ ). Furthermore, since $K_{2}$ is a nontrivial finite simple non-abelian group of orientation-preserving diffeomorphisms of $W_{2}, K_{2}$ cannot leave a torus setwise invariant. Thus the homomorphism is injective, and hence $\operatorname{order}(K)=\operatorname{order}\left(K_{2}\right) \leq m ! \leq d$.

Therefore we can assume that $\Theta$ is empty. If $M$ is not Seifert fibered, then $\operatorname{order}(K)=\operatorname{order}\left(K_{2}\right) \leq d$ by Case 2 of Step 1. So we can further assume that $M$ is Seifert fibered. Now by Waldhausen 22, if $M$ is a closed Haken manifold other than the 3-torus and the double of the twisted $I$-bundle over a Klein bottle, then $M$ has a fibration which is unique up to isotopy. Also, by Ohshika [19, if $M$ is a non-Haken manifold with infinite $\pi_{1}$, then $M$ has a fibration which is unique up to isotopy. Since $M$ is irreducible, if the fibration is unique up to isotopy, then we can apply Meeks and Scott 13 to get a $K_{2}$-invariant fibration. In this case, we can argue as in the end of Case 2 to again conclude that $\operatorname{order}(K) \leq d$.

Thus we an assume that either $M$ is the 3 -torus, $M$ is the double of the twisted $I$-bundle over a Klein bottle, or $M$ has finite fundamental group. Observe that the 3 -torus and the twisted $I$-bundle over a Klein bottle both have flat geometry. By Meeks and Scott [13] any smooth finite group action of a flat manifold preserves the geometric structure. However, by considering the lift of the action to $\mathbb{R}^{3}$ we see that no finite simple non-abelian group can act geometrically and faithfully on either the 3 -torus or the twisted $I$-bundle over a Klein bottle. Thus $M$ cannot be either of these manifolds. 
Finally, suppose that $M$ has finite fundamental group. Now by the proof of the Elliptization Conjecture [16], $M$ has elliptical geometry, and hence by Dinkelbach and Leeb [3] we can assume that $K_{2}$ acts geometrically on $M$. However by the classification of orientation-preserving isometry groups of elliptic 3-manifolds of Kalliongis and Miller [10] and McCullough [12, no finite simple non-abelian geometric group action of an elliptic 3 -manifold has order greater than 60 . Thus $\operatorname{order}\left(K_{2}\right) \leq 60 \leq d$.

Proof of Theorem 1.1. Let $d$ be the number given by Proposition 2.3 for the manifold $M$ and choose $n>d$. Then the alternating group $A_{n}$ is a non-abelian simple group, and by Proposition 2.3 no embedding of any graph $\Gamma$ in $M$ has $\mathrm{TSG}_{+}(\Gamma, M) \cong A_{n}$. Now since $\operatorname{TSG}_{+}(\Gamma, M)$ is either equal to $\operatorname{TSG}(\Gamma, M)$ or is a normal subgroup of $\operatorname{TSG}(\Gamma, M)$ of index 2 , there is no embedding of a graph $\Gamma$ in $M$ such that $\operatorname{TSG}(\Gamma, M) \cong A_{n}$.

\section{Proofs of Theorems 1.2 and 1.3}

In contrast with Theorem 1.1, we prove in Theorem 1.2 that if the manifold $M$ can vary (even among the hyperbolic rational homology spheres), then the collection of topological symmetry groups of embedded graphs is universal. We begin with the following proposition.

Proposition 3.1. Let $M$ be a connected 3-manifold, and let $G$ be a finite group of diffeomorphisms acting freely on $M$. Then there is a graph $\Lambda$ embedded in $M$ such that $\operatorname{TSG}(\Lambda, M) \cong G$.

Proof. Let $n=\operatorname{order}(G)$. If $n=1$ or 2 , we can choose $\Lambda$ to be a single vertex or a single edge in $M$, respectively. Thus we assume that $n>2$.

Let $U, V$, and $W$ be sets of $n$ vertices each. Let $\gamma$ be the abstract graph with vertices in $U \cup V \cup W$ and an edge between a pair of vertices if and only if precisely one of the vertices is in $V$. Then every automorphism of $\gamma$ leaves the set $V$ setwise invariant since the valence of the vertices in $V$ is twice that of the vertices in $U \cup W$. It follows that if an automorphism of $\gamma$ setwise fixes an edge, then it fixes both vertices of that edge.

We embed $\gamma$ in $M$ as follows. Let $u, v$, and $w$ be points in $M$ whose orbits under $G$ are disjoint. Embed the sets $U, V$, and $W$ as the orbits of $u, v$, and $w$ respectively under $G$. We abuse notation and refer to both the abstract and embedded sets of vertices as $U, V$, and $W$. Since $G$ acts freely on $M, G$ induces a faithful action of the abstract graph $\gamma$ such that no non-trivial element of $G$ fixes any vertex or inverts any edge of $\gamma$. Furthermore, the quotient map $\pi: M \rightarrow M / G$ is a covering map and $M / G$ is a 3 -manifold.

Let $\left\{\varepsilon_{1}, \ldots, \varepsilon_{m}\right\}$ consist of one representative from each orbit of the edges of the abstract graph $\gamma$ under $G$, and for each $i$ let $x_{i}$ and $y_{i}$ denote the embedded vertices of $\varepsilon_{i}$. Since $M$ is path connected, for each $i$ we can choose a path $\alpha_{i}$ in $M$ from $x_{i}$ to $y_{i}$ and let $\alpha_{i}^{\prime}=\pi\left(\alpha_{i}\right)$. Since $G$ leaves each of $U, V$, and $W$ setwise invariant, each $\alpha_{i}^{\prime}$ has distinct endpoints. Now, by general position in $M / G$, we can homotop each $\alpha_{i}^{\prime}$ fixing its endpoints to a simple path $\rho_{i}^{\prime}$ such that the interiors of the $\rho_{i}^{\prime}$ are pairwise disjoint and are disjoint from $\pi(V \cup U \cup W)$. For each $i$, let $\rho_{i}$ denote the lift of the path $\rho_{i}^{\prime}$ beginning at $x_{i}$. Then $\rho_{i}$ is a simple path in $M$, and since $\rho_{i}^{\prime}$ is homotopic fixing its endpoints to $\alpha_{i}^{\prime}$, the other endpoint of $\rho_{i}$ is $y_{i}$. For each $i$, embed the abstract edge $\varepsilon_{i}$ as the image of $\rho_{i}$ in $M$. 
Now let $\varepsilon$ be an arbitrary edge of $\gamma$. Since no edge of $\gamma$ is setwise fixed by a non-trivial element of $G$, there is a unique $g \in G$ and $i$ such that $\varepsilon=g\left(\varepsilon_{i}\right)$. Hence we can unambiguously embed $\varepsilon$ as $g\left(\rho_{i}\right)$. Let $\Gamma$ consist of the embedded vertices $V \cup U \cup W$ together with embeddings of the edges of $\gamma$ defined in this way. It follows from our choice of the paths $\rho_{i}^{\prime}$ in $M / G$ that these embedded edges are pairwise disjoint and their interiors are disjoint from the set of vertices $V \cup U \cup W$. Thus $\Gamma$ is indeed an embedding of $\gamma$ in $M$ and is setwise invariant under $G$.

Now let the set $\left\{\rho_{1}, \ldots, \rho_{m}\right\}$ consist of one representative from each orbit of the embedded edges of $\Gamma$ under $G$. We create a new embedded graph $\Lambda$ by adding $i$ vertices of valence 2 to the interior of every edge in the orbit of $\rho_{i}$ in such a way that $G$ leaves $\Lambda$ setwise invariant. Then $G$ induces a faithful action on $\Lambda$, and hence is isomorphic to a subgroup of $\operatorname{TSG}(\Lambda, M)$.

We prove as follows that $G \cong \operatorname{TSG}(\Lambda, M)$. Let $h$ be a homeomorphism of $M$ inducing a non-trivial automorphism of $\Lambda$. Since $\Gamma$ has no vertices of valence $2, h$ leaves $\Gamma$ setwise invariant inducing a non-trivial automorphism of $\Gamma$. Hence there is some edge $e$ of $\Gamma$ such that $h(e) \neq e$. Now $e$ is in the orbit of some $\rho_{i}$ under $G$, and hence as a path in $\Lambda, e$ contains precisely $i$ vertices of valence 2 . Thus $h(e)$ also contains precisely $i$ vertices of valence 2 , and hence is also in the orbit of $\rho_{i}$ under $G$. It follows that for some $g \in G, g(e)=h(e)$. Now $f=g^{-1} h$ is a homeomorphism of $(M, \Gamma)$ taking $e$ to itself, and hence fixing both vertices of $e$ as an edge in $\Gamma$.

Suppose, for the sake of contradiction, that there is some edge $e^{\prime}$ adjacent to $e$ such that $f\left(e^{\prime}\right) \neq e^{\prime}$. Since $f$ fixes both vertices of $e, f$ must fix the vertex $x=e \cap e^{\prime}$. By repeating the above argument with $f\left(e^{\prime}\right)$ instead of $h(e)$, we see that there is a $g_{1} \in G$ such that $g_{1}\left(e^{\prime}\right)=f\left(e^{\prime}\right)$. However, since $G$ acts freely on $M, g_{1}$ cannot fix $x$. Thus $g_{1}\left(e^{\prime}\right)$ has vertices $x$ and $g_{1}(x)$. Since $g_{1}\left(e^{\prime}\right)$ is an edge of $\Gamma$, precisely one of its vertices is contained in $V$. But this is impossible since $g_{1}$ leaves $V$ setwise invariant. Thus $f\left(e^{\prime}\right)=e^{\prime}$, and hence inductively we see that $f$ leaves every edge of $\Gamma$ setwise invariant. Since $f$ cannot interchange the vertices of any edge of $\Gamma, f$ induces the trivial automorphism on $\Gamma$ and hence on $\Lambda$ as well. Thus, $g$ induces the same automorphism as $h$ on $\Lambda$. It follows that $\operatorname{TSG}(\Lambda, M) \cong G$.

Now Theorem 1.2 follows immediately from Proposition 3.1, since Cooper and Long [2] have shown that for every finite group $H$, there is a hyperbolic rational homology 3 -sphere $M$ with a group of diffeomorphisms $G \cong H$ such that $G$ acts freely on $M$.

It was proved in [5] that if a 3 -connected graph $\Gamma$ is embedded in $S^{3}$, then $\mathrm{TSG}_{+}\left(\Gamma, S^{3}\right)$ is isomorphic to a subgroup of the group of orientation-preserving diffeomorphisms Diff $+\left(S^{3}\right)$. We show below that this is not true for all 3-manifolds.

Proof of Theorem 1.3. By the Geometrization Theorem [15, 16, 17, $M$ can be decomposed into geometric pieces. Also, since $M$ is irreducible and not Seifert fibered, $M$ does not admit a circle action. Furthermore, by the Elliptization Theorem [16, a 3-manifold with finite fundamental group is elliptic and hence Seifert fibered. Thus $M$ has infinite $\pi_{1}$, has no circle action, and is irreducible. Hence by Kojima [1] there is a bound on the order of finite groups of diffeomorphisms of $M$. In particular, there is a prime $p>3$ such that $\mathbb{Z}_{p}$ is not a subgroup of Diff $+(M)$. Now it follows from [4] that there is an embedding $\Delta$ of the complete graph $K_{p}$ in the interior of a ball $B$ such that $(B, \Delta)$ has an orientation-preserving diffeomorphism $h$ which induces an automorphism of order $p$ on $\Delta$. Since $h$ is orientation-preserving, 
$h$ is isotopic to the identity on $B$. Thus we can modify $h$ by an isotopy to get a diffeomorphism $g$ of $(B, \Delta)$ such that $g \mid \partial B$ is the identity and $g$ induces an automorphism of order $p$ on $\Delta$. Now we embed $B$ in $M$ and extend $g$ to $M$ by the identity. This gives us an embedding $\Gamma$ of $K_{p}$ in $M$ with $\mathbb{Z}_{p} \leq \operatorname{TSG}_{+}(\Gamma)$. It follows that $\mathrm{TSG}_{+}(\Gamma)$ cannot be isomorphic to any finite subgroup of Diff $+(M)$. Finally, since $p>3, K_{p}$ is 3 -connected.

\section{REFERENCES}

1. F. Bonahon, Geometric structures on 3-manifolds, Handbook of Geometric Topology, 93-164, North-Holland, Amsterdam, 2002. MR1886669 (2003b:57021)

2. D. Cooper and D. Long, Free actions of finite groups on rational homology 3-spheres, Topology Appl. 101 (2000), 143-148. MR.1732066 (2000j:57043)

3. J. Dinkelbach and B. Leeb, Equivariant Ricci flow with surgery and applications to finite group actions on geometric 3-manifolds, Geom. Topol. 13 (2009), 1129-1173. MR2491658 (2011b:53158)

4. E. Flapan, Rigidity of graph symmetries in the 3-sphere, J. Knot Theory Ramifications 4 (1995), 373-388. MR1347360 (96h:57002)

5. E. Flapan, R. Naimi, J. Pommersheim, and H. Tamvakis, Topological symmetry groups of embedded graphs in the 3-sphere, Comment. Math. Helv. 80 (2005), 317-354. MR2142245 (2006i:57006)

6. R. Frucht, Herstellung von Graphen mit vorgegebener abstrakter Gruppe, Compositio Math. 6 (1939), 239-250. MR 1557026

7. A. Hurwitz, Über algebraische Gebilde mit Eindeutigen Transformationen in sich, Math. Annalen 41 (1893), 403-442.

8. W. H. Jaco and P. B. Shalen, Seifert fibred spaces in 3-manifolds, Mem. Amer. Math. Soc. 21 (1979), no. 220. MR539411 (81c:57010)

9. K. Johannson, Homotopy equivalences of 3-manifolds with boundaries, Lecture Notes in Mathematics, 761, Springer, Berlin, 1979. MR551744 (82c:57005)

10. J. Kalliongis and A. Miller, Geometric group actions on lens spaces, Kyungpook Math. J. 42 (2002), 313-344. MR1942194 (2003k:57025)

11. S. Kojima, Bounding finite groups acting on 3-manifolds, Math. Proc. Cambridge Philos. Soc. 96 (1984), 269-281. MR757660 (87b:57014)

12. D. McCullough, Isometries of elliptic 3-manifolds, J. London Math Soc. 65 (2002), 167-182. MR $1875143(2002 \mathrm{j}: 57030)$

13. W. Meeks and P. Scott, Finite group actions on 3-manifolds, Invent. Math. 86 (1986), 287346. MR856847 (88b:57039)

14. E. E. Moise, Geometric Topology in Dimensions 2 and 3, Springer-Verlag, New York, 1977. MR0488059(58:7631)

15. J. W. Morgan and F. T.-H. Fong, Ricci Flow and Geometrization of 3-manifolds, University Lecture Series, 53, American Mathematical Society, Providence, RI, 2010. MR2597148 (2011d:53160)

16. J. Morgan and G. Tian, Ricci flow and the Poincaré conjecture, Clay Mathematics Monographs, 3, American Mathematical Society, Providence, RI; Clay Mathematics Institute, Cambridge, MA, 2007. MR2334563(2008d:57020)

17. J. Morgan and G. Tian, Completion of the proof of the Geometrization Conjecture, arXiv:0809.4040

18. G. Mostow, Strong Rigidity of Locally Symmetric Spaces, Annals of Mathematics Studies, 78, Princeton University Press, Princeton, NJ, 1973. MR.0385004 (52:5874)

19. K. Ohshika, Teichmüller spaces of Seifert fibered manifolds with infinite $\pi_{1}$, Topology Appl. 27 (1987), 75-93. MR910495 (88k:57014)

20. J. Simon, Topological chirality of certain molecules, Topology 25 (1986), 229-235. MR.837623 $(87 \mathrm{~m}: 57007)$

21. W. Thurston, Three-dimensional manifolds, Kleinian groups and hyperbolic geometry, Bull. Amer. Math. Soc. 6 (1982), 357-381. MR648524(83h:57019)

22. F. Waldhausen, Eine Klasse von 3-dimensionalen Mannigfaltigkeiten II, Invent. Math 4 (1967), 87-117. MR0235576(38:3880) 
23. F. Waldhausen, On irreducible 3-manifolds which are sufficiently large, Ann. of Math. (2) 87 (1968), 56-88. MR0224099(36:7146)

24. B. Zimmerman, On finite simple groups acting on homology 3-spheres, Topology Appl. 125 (2002), 199-202. MR1933571 (2003m:57041)

Department of Mathematics, Pomona College, Claremont, California 91711

E-mail address: eflapan@pomona.edu

Department of Mathematics, University of Maryland, College Park, Maryland 20742

E-mail address: harryt@math.umd.edu 